\title{
Wood Species and Culture Age Affect Zone Line Production of Xylaria polymorpha
}

\author{
Sara C. Robinson* and Peter E. Laks \\ Michigan Technological University, 1400 Townsend Drive, Hougton, MI 49931-1295, USA
}

\begin{abstract}
Three pure cultures of Xylaria polymorpha were isolated from fruiting bodies at yearly intervals over two years and maintained on $2 \%$ malt agar plates at room temperature. Immediately after isolation of the third culture, the cultures were inoculated onto sugar maple (Acer saccharum), aspen (Populus tremuloides), birch (Betula alleghaniensis), and basswood (Tilia americana) $14 \mathrm{~mm}$ cubes and incubated for 10 weeks in jars containing vermiculite. More zone lines were produced on aspen and sugar maple than on yellow birch or basswood. Increasing culture age generally caused a decrease in zone line production; however the effect was only statistically significant in sugar maple. The results indicate that aspen is preferable for zone line production with $X$. polymorpha, as both external and internal zone lines occur on this wood species, and zone line production remains high despite the age of the culture.
\end{abstract}

Keywords: Spalting, Xylaria polymorpha, zone lines.

\section{INTRODUCTION}

Zone lines can be produced by a wide variety of basidiomycete fungi and some ascomycete fungi. They often appear macroscopically as thin, dark lines that delineate patches of fungal colonization. Zone lines are generally produced by a fungus in response to antagonism from another fungus, from somatic incompatibility within the hyphal mass of a single fungus, or from colonization of a non-decay fungus within a zone of undecayed wood [1-5].

A wide variety of wood decay fungi produce zone lines via interspecific antagonism [3, 6, 7]. However, only a small number of fungi have been documented that produce zone lines by themselves in pure culture $[1,2,8]$. Xylaria polymorpha (Pers.) Grev. is one of the most well known intraspecific antagonistic fungi. It is a white rot ascomycete fungus that grows on a variety of hardwoods, including beech (Fagus), maple (Acer), and oak (Quercus) [9, 10], and has been studied for its zone line production since 1933 [1]. In pure culture, the zone lines of $X$. polymorpha are most likely produced due to intraspecific antagonism - where each zone line delineates a genetically distinct individual [4].

Although zone lines usually indicate wood decay, their presence can add value to lumber. The production of zone lines is particularly important for spalted wood production. Spalting is any color formed on wood due to fungus colonization, whether penetrating or remaining only on the surface. Zone lines are the most well known type of spalting, and are relatively easy to induce under laboratory conditions [7]. Although $X$. polymorpha does not colonize wood as quickly as zone line producing basidiomycete fungi [7], this fungus has been increasingly used in laboratory spalting due to its ability to produce zone lines without the presence of another fungus.

*Address correspondence to this author at the Michigan Technological University, 1400 Townsend Drive, Hougton, MI 49931-1295, USA;

Tel: 416-879-8826; E-mail: scrobins@mtu.edu
As commercial interest in spalted wood increases, it is necessary to understand not just which wood species are most quickly decayed by a specific fungus, but to discover which wood species are most quickly spalted, preferably without an associated increase in decay. The Ohio DNR states that birch (Betula), beech, and maple are the most commonly spalted woods [11]; however a recent study on preferred hosts of Chlorociboria spp. indicates that aspen (Populus) may also readily spalt [12]. An additional concern in regards to laboratory spalting is the effectiveness of longterm cultures. Some fungi are known to lose virulence over time when stored under certain conditions, while others appear to be unaffected [13, 14]. In many cases colony diameter is used as a measure of culture viability $[15,16]$, however viability of spalting fungi requires pigment and/or zone line production to be maintained, not colony size. Hence, repeatable laboratory experiments require fungi whose spalting ability does not decrease over time.

The objectives of this research were to (1) identify a preferred host for $X$. polymorpha and (2) determine if zone line production decreased over time due to culture storage. Selected wood species were chosen due to their low extractive content and past utilization with spalting fungi $[7$, $12,17]$.

\section{EXPERIMENTAL}

Three pure cultures of $X$. polymorpha were collected, once per year, over the course of two years. The culture tissue was collected from the same sugar maple stump and the same grouping of fruiting bodies each year (July 2007, August 2008, August 2009 in Alberta, MI on an Acer saccharum stump) in an effort to minimize genetic variability. Each year, a section of the fruiting body was removed from the stump, opened in a sterile laminar flow hood, and tissue sections removed with sterile tweezers. The tissue cultures were plated onto $2 \%$ malt agar plates immediately after removal. After collection and isolation, the 
cultures were stored on $2 \%$ malt agar plates $(95 \times 15 \mathrm{~mm}$ Petri plates) at room temperature $(22 \mathrm{C})$. Cultures were transferred to fresh plates at three month intervals to maintain active growth.

Four wood species were chosen for inoculation: trembling aspen (Populus tremuloides Michx., 12\% SG (specific gravity based upon dry mass and volume at $12 \%$ moisture content) $=0.45,12 \% \mathrm{MC}$ (moisture content) density (mass at $12 \% \mathrm{MC}$ per volume at $12 \% \mathrm{MC})=483 \mathrm{~kg} / \mathrm{m}^{3}$ ), sugar maple (Acer saccharum Marsh., $12 \% \quad \mathrm{SG}=0.66, \quad 12 \% \quad \mathrm{MC}$ density $=710 \mathrm{~kg} / \mathrm{m}^{3}$ ), yellow birch (Betula alleghaniensis Britt., $12 \% \mathrm{SG}=0.59,12 \% \mathrm{MC}$ density $=636 \mathrm{~kg} / \mathrm{m}^{3}$ ), and basswood (Tilia americana L., $12 \% \mathrm{SG}=0.41,12 \% \mathrm{MC}$ density $=430 \mathrm{~kg} / \mathrm{m}^{3}$ ). The wood used was selected for the absence of visible surface defects or spalting. Thirty $14 \mathrm{~mm}$ blocks of each species were cut and tested with each fungal isolate (30 replicates per wood species per fungus), giving a total of 360 tested blocks.

Decay jars $(4 \times 4 \times 12 \mathrm{~cm}$ flint jars) containing vermiculite were prepared identically to the procedure described in Robinson et al [17]. The jars were autoclaved for 30 minutes, and then allowed to cool overnight in a laminar flow hood prior to inoculation.

Before inoculation, the wood blocks were oven dried at $40 \mathrm{C}$ for 24 hours and weighed. They were then steam sterilized for 30 minutes, allowed to cool, and placed in the decay jars. Two blocks were placed in each jar, with a strip of inoculum (roughly $2 \times 2 \mathrm{~cm}$ ) sandwiched between the two transverse planes. Blocks were oriented so that the transverse faces ran parallel to the length of the jars. Each set of two blocks was pushed into the vermiculite, so that both were completely covered. No feeder strips were used.

Jars were incubated in a humidity and temperature controlled room $(27 \mathrm{C} \pm 2 \mathrm{C}, 80 \% \pm 5 \%$ relative humidity) for 10 weeks. After incubation, the blocks were removed from their jars, scrubbed and washed to remove residual vermiculite and mycelium, dried overnight at $40 \mathrm{C}$, and reweighed to determine weight loss. After drying, the external face of each block with the most spalting was scanned at 2400 dpi using an Epson Perfection V100 photo scanner. Blocks were then cut in half to expose an internal radial face; the side with the most spalting was again scanned.

Zone line amounts were analyzed using the protocol developed for Scion Image Software [18]. All data were analyzed at $\alpha=0.05$ using a two-way analysis of variance (ANOVA) with wood species and isolate as independent variables. If the interaction term was significant, a means model was run to determine the location of the significant differences. If the interaction term was not significant, a Tukey HSD test was run to determine the location of the significant differences. All percentage data were transformed using arcsine square root to meet ANOVA assumptions of error term variance and normality. The statistical procedures were performed with SAS, version 9.2 (SAS 2009 system for Windows).

\section{RESULTS}

No significant weight loss occurred with any of the wood species, regardless of the isolate used. Wood species $(\mathrm{P}<0.0001)$, isolate $(\mathrm{P}=0.0029)$, and the interaction between the wood species and isolate $(\mathrm{P}=0.0021)$ were significant. The means model performed on the interaction term was also highly significant $(\mathrm{P}<0.0001)$. Results from the 2-way ANOVA on internal spalting found that wood species $(\mathrm{P}=0.0010)$ and isolate $(\mathrm{P}=0.002)$ were significant.

Externally, zone line production was generally consistent between isolates for each wood species (Fig. 1). In general, aspen and sugar maple had the most zone lines, while basswood had the least (Fig. 2). The amount of zone lines produced by each isolate did not differ significantly $(\alpha=0.05)$ for any species, with the exception of sugar maple. The 2007 $X$. polymorpha isolate (SR001) produced significantly fewer zone lines on sugar maple than the 2008 or 2009 isolates.

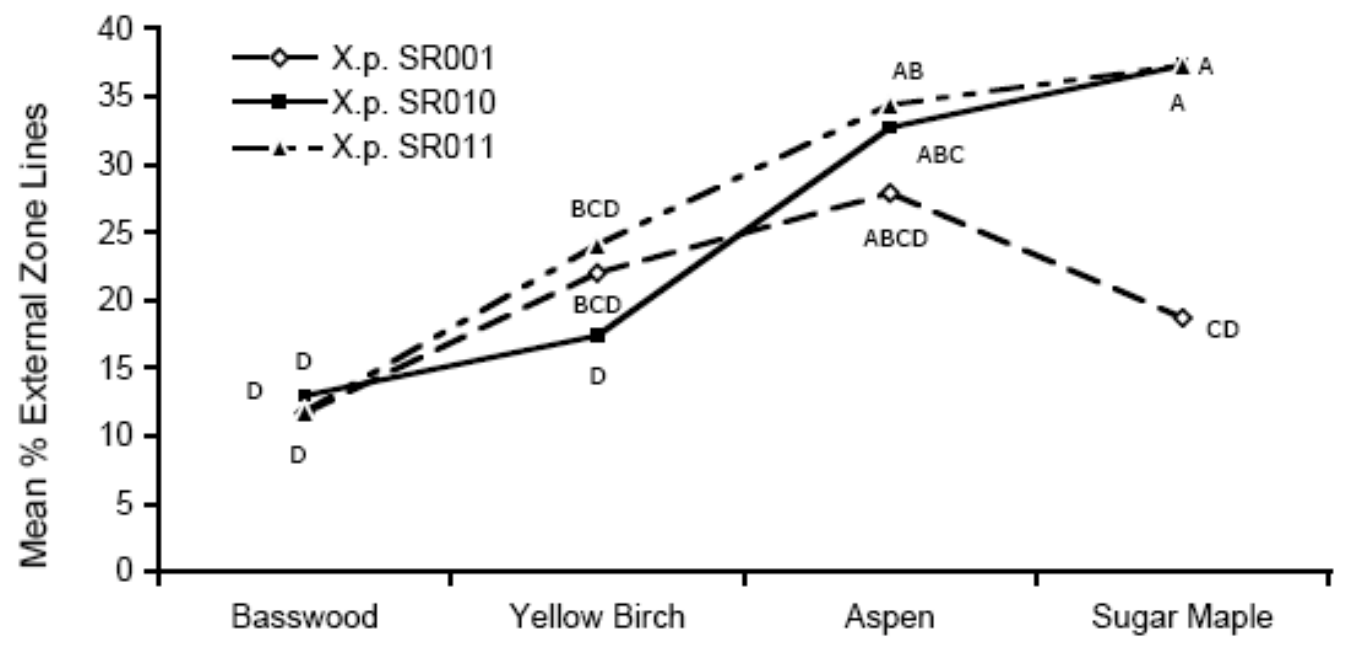

Wood Species

Fig. (1). Mean percent external zone lines per block formed on four different wood species with Xylaria polymorpha isolates. Different letters represent significant differences $(\alpha=0.05)$. Graph shows transformed data values (data transformed using arcsine square root). 


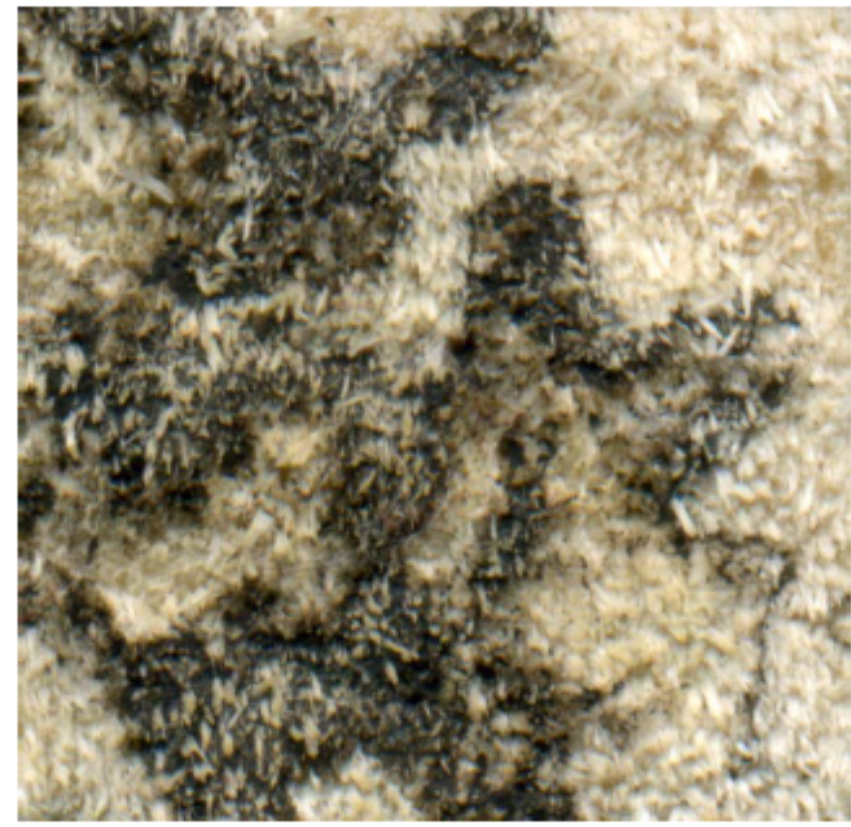

A

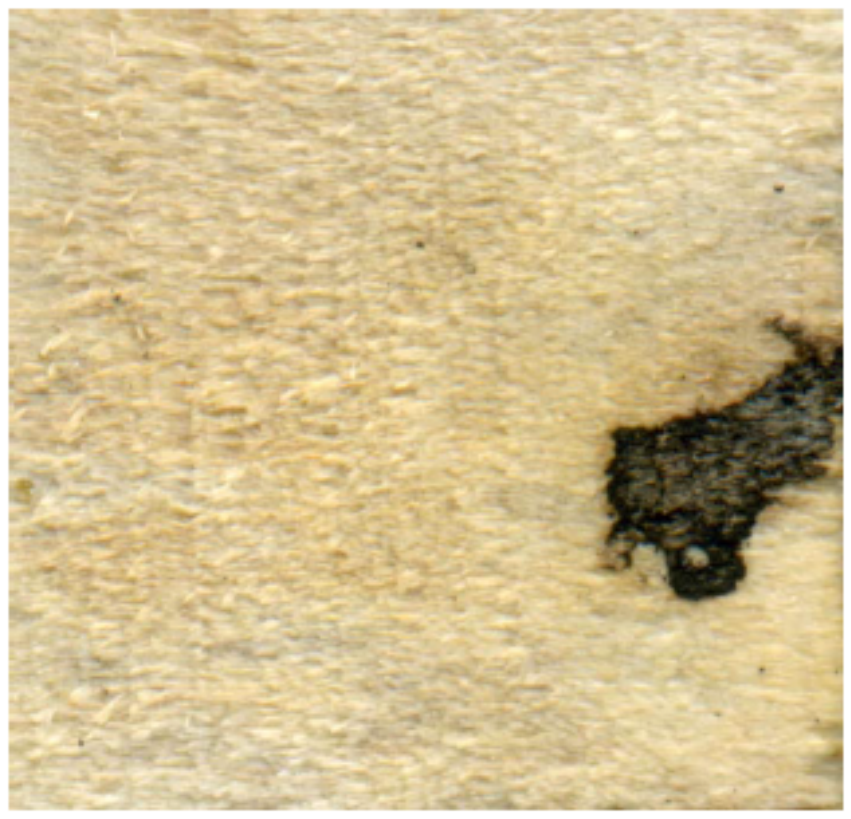

C

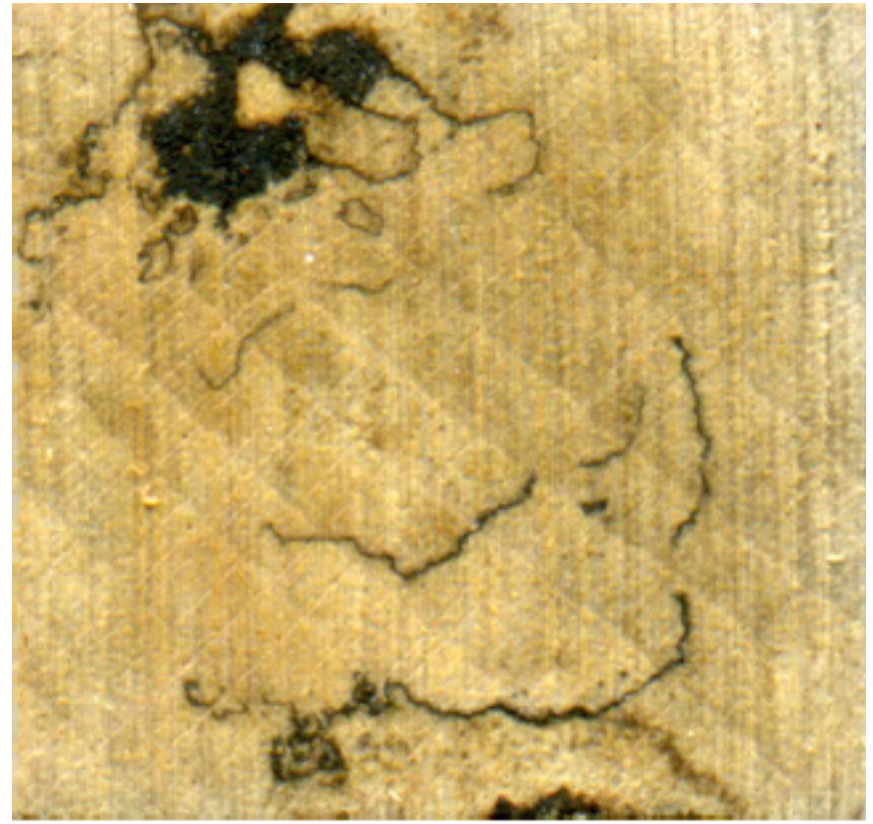

B

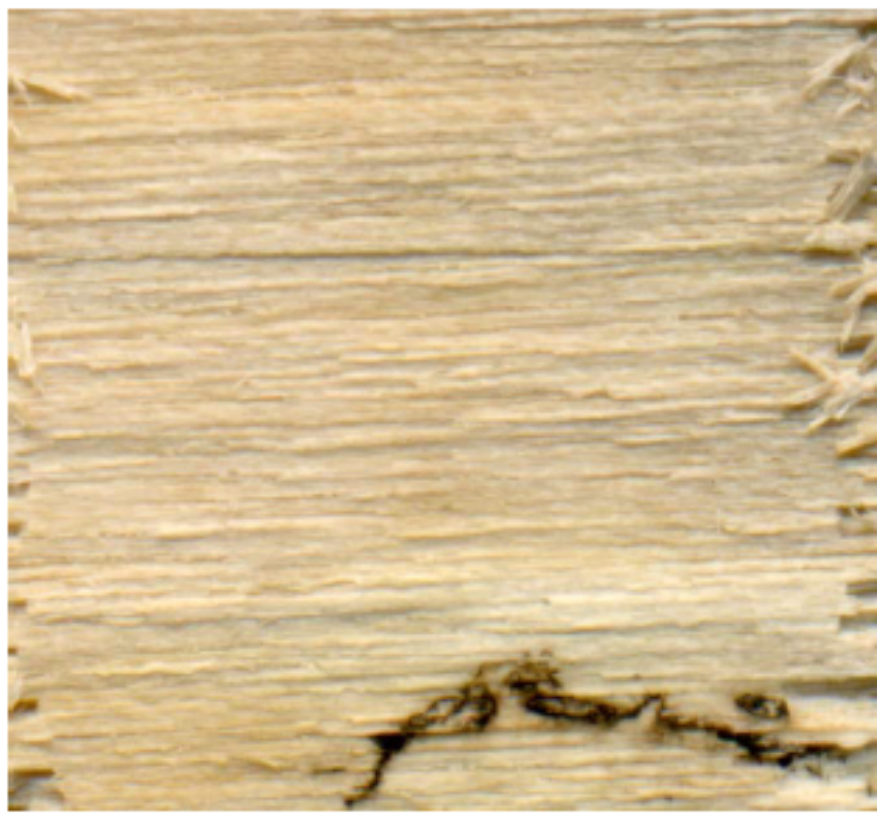

$\mathrm{D}$

Fig. (2). Typical external zone line production on (A) aspen, (B) sugar maple, (C) basswood, and (D) yellow birch.

Zone lines seldom occurred internally. There were significantly more internal zone lines in aspen $(\alpha=0.05)$ than any other wood species; however, the amount of zone lines in all species was very low (average of $1.8 \%$ for aspen, and less than $1 \%$ for all other wood species). Isolate SR011 produced significantly more zone lines than either of the other two isolates $(\alpha=0.05)$, however, the amounts were also very low (average of $1.5 \%$ for SR011, and less than $1 \%$ for SR001 and SR010).

\section{DISCUSSION}

The apparent preference of X. polymorpha for aspen and sugar maple is consistent with similar work done with
Chlorociboria spp. testing [12]. Although both $X$. polymorpha and Chlorociboria spp. are Ascomycetes, $X$. polymorpha has the ability to cause white rot decay [19]. It is probable that $X$. polymorpha behaves similar to a stain fungus when wood sugars are readily available, and then reverts to lignin and holocellulose degradation when simple sugars become scarce. This may explain why it has similar wood preferences to Chlorociboria spp.

It is unknown why $X$. polymorpha appears to preferentially colonize aspen and sugar maple. Of the four wood species tested, none have a high or even moderate decay resistance, and all have the same general pore distribution and size. Chemically, there are no large differences among 
the four wood species, with aspen and basswood having a very similar cellulose, hemicellulose, and lignin composition [20]. Since both Chlorociboria spp. and X. polymorpha show a similar wood species preference, it is possible that this preference may have less to do with requirements of a specific fungus, and more to do with some unknown differences in wood chemical composition or microanatomy.

The results from this study indicate that $X$. polymorpha slowly decreases its zone line production over laboratory storage time; however, this decrease is only significant after two years and only when the fungus is inoculated onto sugar maple. The reason for the decline in zone line amounts on sugar maple with the oldest $X$. polymorpha isolate is unknown. It seems unusual that the 2007 isolate would perform poorly on sugar maple, but not on any other wood. We hypothesize that the subtle changes in X. polymorpha over time when kept in culture are somehow exacerbated by an unknown characteristic of sugar maple, and that these changes do not begin to affect zone line production until after two years of storage.

It is possible that other culture storage techniques (e.g. lyophilization) could be utilized to maintain the zone line production in X. polymorpha. However, a less technological approach is required. Spalting research relates directly to wood products applications, and it is therefore necessary to utilize culture techniques that are readily accessible to a large number of people.

Although aspen and sugar maple both contained more zone lines than yellow birch or basswood, the sharp decrease in zone line production with the 2007 isolate and the occurrence of internal zone lines indicates that aspen may be the most preferable wood species for zone line production with $X$. polymorpha. Previous research has shown that aspen (and to a lesser extent, sugar maple) is also the preferred wood species of Chlorociboria spp. The data from this research and from Robinson and Laks [12] indicate that aspen and maple allow for greater amounts of spalting than yellow birch, which was previously regarded as a commonly spalted wood [11]. This research also suggests that some wood species show greater amounts of spalting than others, regardless of which fungus is used as inoculum, and that increasing isolate age may decrease zone line production on sugar maple.

The apparent preference of $X$. polymorpha and Chlorociboria spp. for aspen and sugar maple is important to the development of commercially produced spalted wood. Most research-oriented and commercial spalting work has only utilized sugar maple as a medium. The use of aspen creates an alternative spalted wood choice for consumers, allows for the use of older fungal cultures for inoculation, and allows for increased green stain from Chlorociboria spp. If additional research finds that other spalting fungi also prefer aspen and sugar maple, then the use of these wood species should allow for large amounts, and even multiple types of spalting within a single piece of wood. In addition, utilization of a limited number of wood species should decrease potential variability in incubation time, producing a more uniform product.

\section{REFERENCES}

[1] Campbell AH. Zone lines in plant tissues.1: the black lines formed by Xylaria polymorpha (Pers.) Grev. in hardwoods. Ann Appl Biol 1933; 20: 123-45.

[2] Campbell AH. Zone lines in plant tissues. II: the black lines formed by Armillaria mellea. Ann Appl Biol 1934; 21(1): 1-22.

[3] Rayner ADM. The challenge of individualistic mycelium. Mycologia 1991; 83(1): 48-71.

[4] Rayner ADM, Todd NK. Intraspecific antagonism in natural populations of wood-decaying basidiomycetes. J Gen Microbiol 1977; 103: 85-90.

[5] Rayner ADM, and Todd NK. Population and community structure and dynamics of fungi in decaying wood. Adv Bot Res 1979; 7 : 333-420.

[6] Phillips LW. The nature of spalted wood: analysis of zone line formation between six white rot fungi. Department of Industrial Education. Provo, UT, Brigham Young University. Masters of Science; 1987.

[7] Robinson SC, Richter DL, Laks PE. Colonization of sugar maple by spalting fungi. Forest Prod J 2007; 57(4): 24-32.

[8] Williams END, Todd NK, Rayner ADM. Spatial development of populations of Coriolus versicolor. New Phytol 1981; 89(2): 30719.

[9] Cartwright G, St. K, Findlay, WPK. Decay of timber and its prevention. New York: Chem. Publ. Co.; 1950.

[10] Anonymous. Index of plant diseases in the United States. USDA Agriculture Hand book No. 165, 1960.

[11] Ohio DNR. The Spalted Wood. [cited Oct 2009]. Available from: http://www.ohiodnr.com/tabid/5255/Default.aspx

[12] Robinson SC, Laks PE. Wood species affects colonization rates of Chlorociboria sp. Int Biodeterior Biodegradation 2010b; (in press).

[13] Honegger R. The impact of different long-term storage conditions on the viability of lichen-forming Ascomycetes and their green algal photobiont, Trebouxia spp. Plant Biol 2003; 5: 324-30.

[14] Pasarell L, McGinnis MR. Viability of fungal cultures maintained at -70 degrees. C. J Clin Microbiol 1992; 30(4): 1000-4.

[15] Hwang S. Effects of ultra-low temperatures on the viability of selected fungus strains. Mycologia 1960; 52(3): 527-9.

[16] Richter DL. Revival of saprotrophic and mycorrhizal basidiomycete cultures after 20 years in cold storage sterile water. Can J Microbiol 2008; 54(8): 595-9.

[17] Robinson SC, Laks PE, Turnquist EJ. A method for digital color analysis of spalted wood using Scion Image software. Materials [serial on the internet]. [cited 2009 October]; 2009a; 2(1): 62-75. Available from: http:/www.mdpi.com/1996-1944/2/1/62/pdf

[18] Robinson SC, Richter DL, Laks PE. Effects of substrate on laboratory spalting of sugar maple. Holzforschung 2009b; 63: 4915.

[19] Liers C, Ullrich R, Steffen KT, Hatakka A, Hofrichter M. Mineralization of ${ }^{14} \mathrm{C}$-labelled synthetic lignin and extracellular enzyme activities of the wood-colonizing ascomycetes Xylaria hypoxylon and Xylaria polymorpha. Appl Microb Cell Physiol 2005; 69(5): 573-9.

[20] Pettersen RC. The chemical composition of wood, Chapter 2. In: Rowell RM, Ed. The chemistry of solid wood. New York: An American Chemical Society Publication 1984. 